\title{
The impact of Arterial Pulse Impedance Artifact (APIA) on test-retest reliability of quantitative $\mathrm{EEG}$
}

\author{
Gerald Ulrich ${ }^{1}$, Willi Schlosser ${ }^{1}$ and Georg Juckel ${ }^{2,3^{*}}$
}

\author{
* Correspondence: \\ georg.juckel@lwl.org \\ 2Department of Psychiatry, \\ Ruhr-University Bochum, LWL \\ University Hospital, Bochum, \\ Germany \\ ${ }^{3}$ Department of Psychiatry, \\ Psychotherapy and Preventive \\ Medicine, LWL University Hospital \\ of the Ruhr University Bochum, \\ Alexandrinenstr.1, 44791 Bochum, \\ Germany \\ Full list of author information is \\ available at the end of the article
}

\begin{abstract}
Background: We report on a biological artifact that is unknown in the EEG-literature, presumably due to its invisibility in the unfiltered raw EEG. On account of its probable electrogenesis, we discuss Arterial Pulse Impedance Artifact (APIA). One may also call it a virtual artifact because it only becomes manifest as the averaged mean value of power spectra from consecutive $2 \mathrm{~s}$ epochs across a total length of 5 min (150 epochs).

Presentation of the hypothesis: APIA has a cardiac origin, mediated by the superficial head arteries. The arterial pulse induces rhythmic changes in the electrical impedance between the cortical generator inside the skull and the recording electrode that is outside.

Testing the hypothesis: APIA will only show up in the EEG if an electrode is fixed very close to the artery. APIA vanishes upon minimal shifting of the corresponding electrode. Detectability in the FFT-spectrogram is due to an amplification effect.

Implications of the hypothesis: The only possibility to avoid this artifact consists in excluding the APIA-prone spectral components below $2.0 \mathrm{~Hz}$ from any further quantification procedure. The importance of this artifact consists in its possible interference with test-retest reliability, for EEG practice and research.
\end{abstract}

Keywords: APIA (Arterial Pulse Impedance Artifact), Steep peaks up to $2 \mathrm{~Hz}$, "Invisible in the "raw EEG", Cardiac origin

\section{Background}

One major factor persistently hampering the development of a psychophysiologic EEG is its deficient test-retest reliability. Its center stage is occupied by a biological artifact that has not previously been described in the literature. Remarkably, this artifact seems to have gone unrecognized by all those who have performed FFT- spectral analysis since the introduction of QEEG. Superficially speaking the main reason should be the invisibility of this artifact within the raw-EEG which is by no means rare.

\section{Presentation of the hypothesis}

When we first called attention to this phenomenon [1] using the acronym PIA (Pulse Impedance Artifact), the response was incomprehension. Later on, we spoke of APIA (Arterial Pulse Impedance Artifact) in order to emphasize its bioelectrical source [2].

(c) 2016 The Author(s). Open Access This article is distributed under the terms of the Creative Commons Attribution 4.0 International License (http://creativecommons.org/licenses/by/4.0/), which permits unrestricted use, distribution, and reproduction in any medium, provided you give appropriate credit to the original author(s) and the source, provide a link to the Creative Commons license, and indicate if changes were made. The Creative Commons Public Domain Dedication waiver (http://creativecommons.org/ publicdomain/zero/1.0/) applies to the data made available in this article, unless otherwise stated. 
There are certain biological artifacts occurring inevitably. In the low frequency range (up to $2.0 \mathrm{~Hz}$ as a rule), they are due to spontaneous blinks of the lids, movements of the ocular bulbi, swallowing or ECG. Conventionally, artifact elimination consists in "eye-ball editing" of the Spontaneous Resting EEG (SR-EEG). A major drawback of this procedure is the unavoidable adulteration of the time-course, which is equivalent to the EEG's dynamic information. As already emphasized introductorily, the most substantial objection against visuo-morphologically artifact editing is the up to now generally unknown considerable amount of APIA because of its invisibility in the raw-EEG [2,3]. If one considers the dynamic information contained in the undistorted time-structure as important, there exists no other corrective than to exclude the whole APIA-prone FFTspectral range from further calculations.

\section{Testing the hypothesis}

Since our aim consisted in the verification of eventual APIAs and not in its elimination we are deliberately resigning with common pre-processing filtering. This is also true for Independent Component Analysis (ICA), a method of multivariate statistics that may render an EEG to be both false and false-negative artifact-contaminated with regard to APIA.

Figures 1, 2, 3 and 4 show the averaged FFT spectra of normal SR-EEG recordings and the APIA revealed by the FFT. In the first recording (I) very steep delta peaks at T4-A2 and F8-A2 but also with somewhat lower amplitude as well as dwindling harmonics in O1-A1, F7-A1, and T3-A1 are depicted. In the following recordings (II or II-VI) there are steep peaks with distinctly lower amplitudes only to be seen in F7-A1 and F8-A2.

This special type of artifact is the most vicious, because it cannot be circumvented by conventional visual editing. There is no other possibility to eliminate APIAs than the exclusion of the APIA-prone spectral components from further calculation procedures, i.e. the slow frequencies below $2.0 \mathrm{~Hz}$. APIA results from a certain topographical relation between electrodes and the superficial head arteries. This relation cannot be influenced by the recording technique, for example the international 10-20 scheme or by taking any preventive measure, whatsoever. Otherwise, APIA cannot be tolerated because it adulterates the spatio-temporal characteristic and thus the dynamics of the SREEG. APIA is characterized by the peculiar coincidence of some observational facts which are compiled as follows.

\section{APIA: The coincident features}

The prerequisite for the visual manifestation of the virtual APIA is averaging across short-epoch FFTs (2s each). A considerable ample number of very low voltage potentials - about 150 - provide for an amplification effect and thus detectability.

I. The paramount and most confident feature for verification are the needle-like steep FFT-spectrographic peaks within the 1.0 to $2.0 \mathrm{~Hz}$ range.

II. The very different amplitudes of the FFT-spectrographic peaks are invisible in the raw EEG

III. In a minority of cases appear multiple peaks within the APIA-prone frequency range 


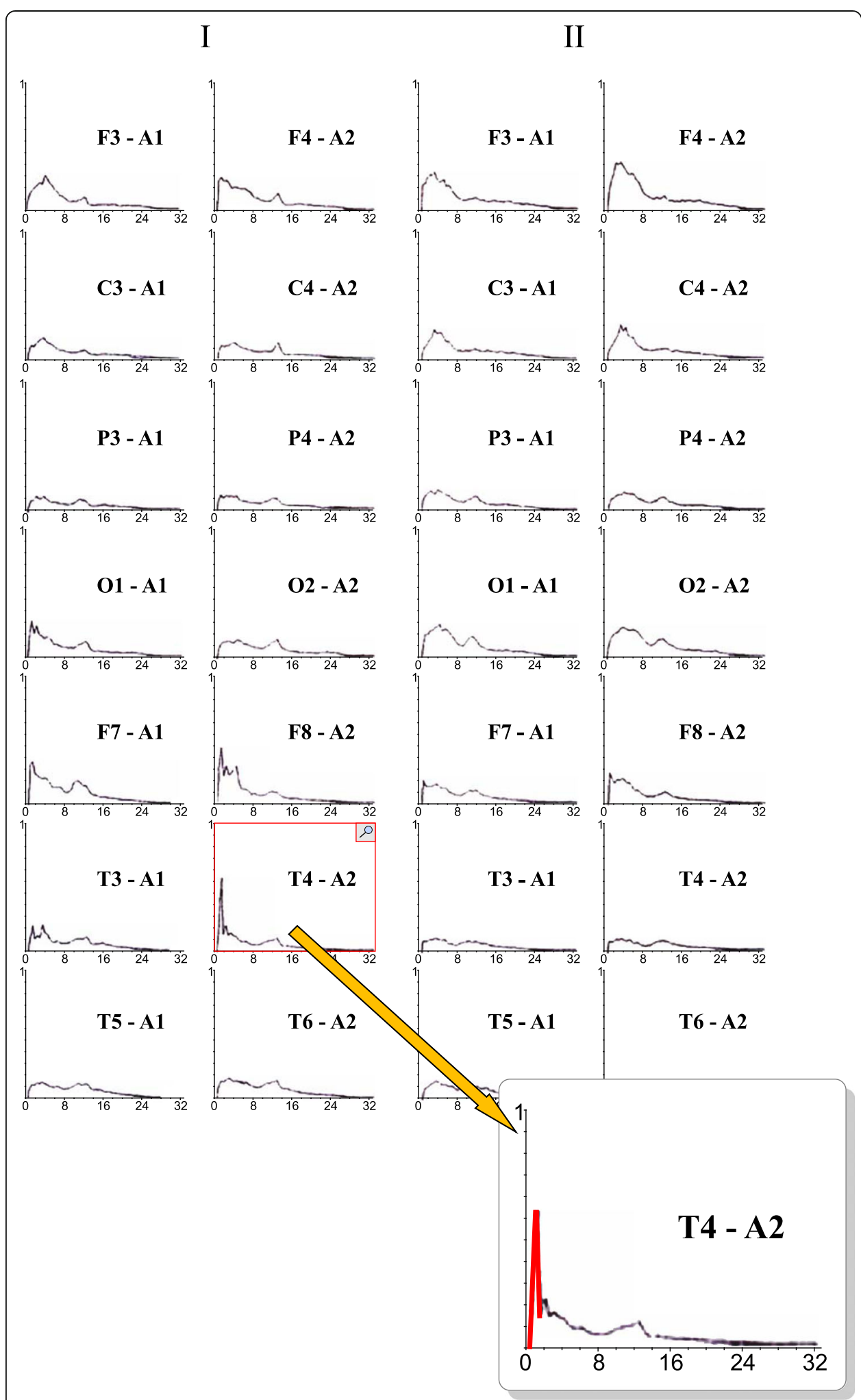

Fig. 1 (See legend on next page.) 
(See figure on previous page.)

Fig. 1 Mean power spectra as calculated from the last 150 consecutive $2 \mathrm{~s}$ epochs (5 min) with ipsilateral reference $(\mathrm{A} 1, \mathrm{~A} 2)$ and $\mathrm{Pz}$ as ground electrode. A ground electrode is needed to serve as a common reference point for all voltages in the system or with respect to collect the grounding of the potentials of an EEG system. Subject: healthy male, 31 years of age; Fig. 1 shows the ipsilateral power spectra with an interval of 4 weeks (I: 01/ 02/08 and II: 02/03/08). Please note channels T4-A2 and F8-A2. Especially the former shows a right-hemispheric needle-like steep peak within the slow Delta-range. A similar peak but less distinct can be observed in the right temporo-frontal region over F8. Notably is the impoverished Alpha peak as an indicator of a desynchronized low voltage EEG to be observed with 7-10\% of adults with an intact brain function

IV. The APIA typically prefer temporal regions, especially F7, F8, T3, T4, T5 and T6; less commonly, regions $\mathrm{O} 1$ and $\mathrm{O} 2$

V. The higher the APIA-amplitudes, the more probable the appearance of damped harmonics

VI. There is no relationship between number and placement of electrodes (10-20 placement of the electrodes) and the number of APIAs.

VII. APIA are not reproducible, being independent on the interval between successive recordings.

\section{Implications of the hypothesis}

It is a well substantiated fact that blood has far better electrical conductivity than water or organic tissue such as bone, muscle or fat because of a higher concentration of electrolytes. As a result, the impedance between the electrodes, fixed on the scalp and the surface of the brain beneath the bony skull will decrease during the phase of systolic blood filling of the superficial head arteries (Fig. 5) and will increase during the subsequent diastolic phase. Thus, the arterial pulse induces rhythmical changes in electrical impedance between the cortical generator inside the skull and the recording electrode outside. This will show up in the EEG if a recording electrode is fixed over or very close to the artery. The impedance variations were definitely not induced by "rhythmic" electrode movements! With a mean heart rate of $60-120$ beats/min, we have reason to expect an empirically observable variation of the impedance value at a frequency of $1-$ $2 \mathrm{~Hz}$ to characterize the typically steep peak of the APIA phenomenon.

APIA vanishes upon minimal shifting of the respective electrodes. As already depicted, Figs. 1, 2, 3 and 4 show more or less typical APIAs with steep peaks in the range below or equal to $2.0 \mathrm{~Hz}$ and implied harmonics.

A cardiac origin can be stated for the virtual artifact mediated by the superficial head arteries. APIA has to be distinguished from the well-known interspersal of the QRScomplexes generated by the electrical activity of the heart and recorded by the electrocardiogram (ECG). This type of EEG artifact may generally be recognized by visual inspection, allowing localized inferences about the position of the electrical heart-axis. Though the frequency range of the QRS-complex partially overlaps with APIA which also applies for blinks and other eye movements they may easily be distinguished from APIA on account of the typical steep-needle-like peaks in the very low frequency range. Furthermore, subjects who show an interspersal of QRS-complexes in their raw EEG will also exhibit them with repeated recordings. A specific distinguishing mark between APIA and the QRS-complexes is the local preference of the former with its systematic bias for the temporal regions. The different focal areas of APIAs and QRS-complexes may be corroborated by Hjorth's source derivations [4], when all is said and done. 


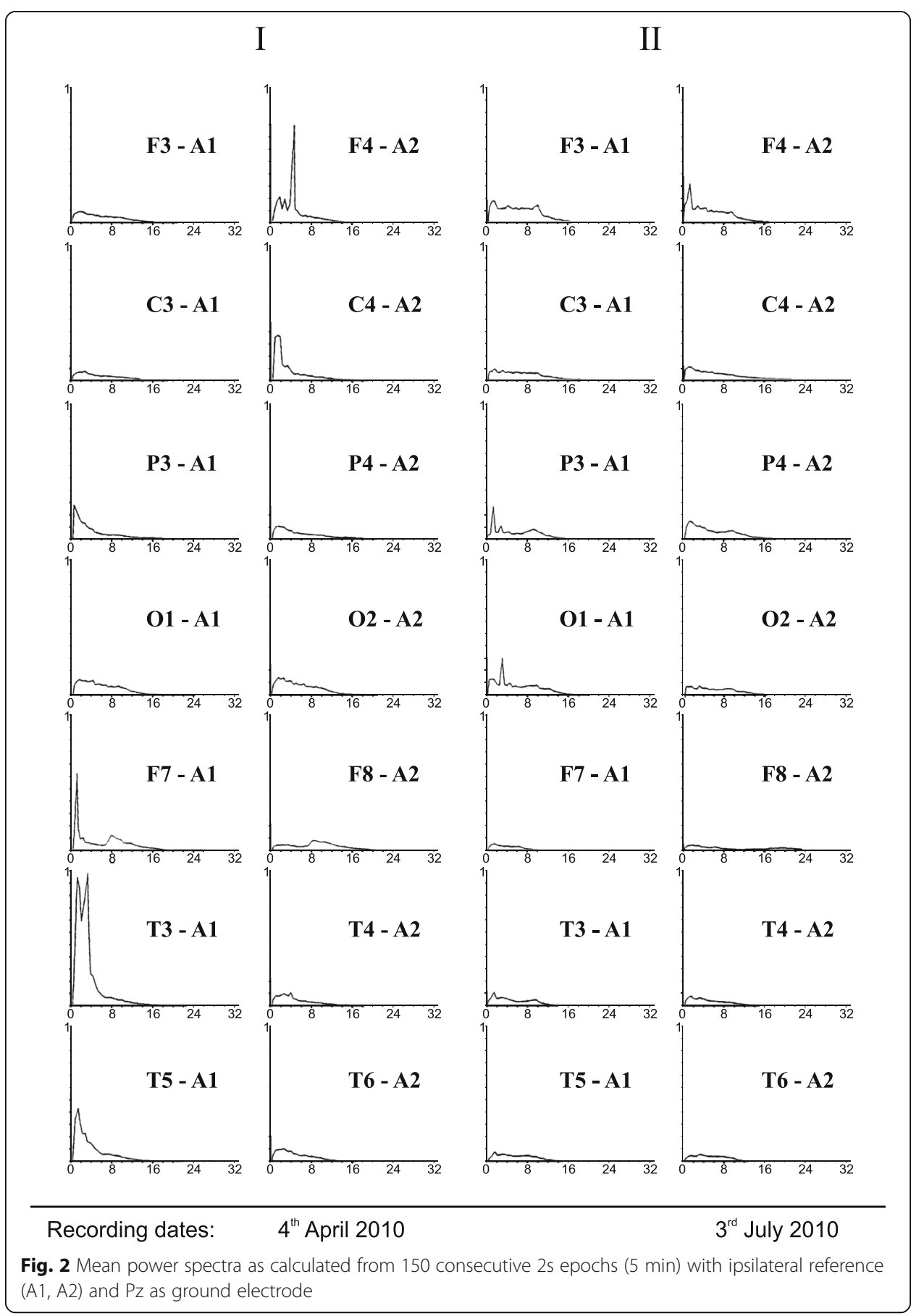

The Figs. 2, 3 and 4 on the next pages show examples of APIA. Electrodes with APIA-typical morphology are highlighted in bold typeface, with less typical morphology in normal typeface. 


\begin{tabular}{cc}
$\begin{array}{c}\text { Number of } \\
\text { recording }\end{array}$ & $\begin{array}{c}\text { APIA } \\
\text { (electrodes) }\end{array}$ \\
\hline I & F4/C4/P3/F7/T3/T5 \\
II & F4/P3/O1 \\
III & F3/C4 /P3/P4/O1/ F8 \\
IV & none \\
V & F3/C3/F8/F7 \\
VI & none
\end{tabular}

The morphology of the phenomenon to be addressed as APIA spans the entire range of variation. F4 (I) triphasic peaks with steep peaks $1-2 \mathrm{~Hz}$ with very different amplitudes, beginning with a $1-2 \mathrm{~Hz}$ of relatively low amplitude, followed by a slightly lower peak of $2-3 \mathrm{~Hz}$ and a steep, higher $3-4 \mathrm{~Hz}$ needle-like peak. In the same recording, five additional APIAs may be detected. On C4, P3, F7, T5 monophasic low peaks may be detected in the slow Delta range; from these only $\mathbf{F} 7$ shows the prototypical needle-like high peak which is similar to the T4 in Fig. 1. A biphasic peak with large amplitude whereby the first peak shows up at $2 \mathrm{~Hz}$ and the second set of 3-4 peaks occurs with T3. In the second recording (II) there occur monophasic APIAs with F4, P3 and O1 with a frequency of 2-3Hz; on account of their low and steep appearance they resemble epileptic spikes. In the third recording (III), F3 shows a prototypical APIA with a needle-like high amplitude morphology and lower slow peaks with C4, P3, P4, O1, and F8. No APIA can be found with the fourth recording (IV). With recording V APIA is implied in the regions F3, C3, F7 and F8 and with recording VI no APIA may be discovered. Remarkably, the subjects, who were chosen as examples for APIA, exhibit only a scant Alpha amount, seeming to belong to the sample of normal variants with low-voltage EEGs that do not show brain dysfunction. But any nexus can be excluded because also APIAs may be demonstrated in subjects with excellent Alpha basic rhythm.

Eventually, by visual inspection or by brain-mapping a distinction can be performed. The detectability is due to an amplification-effect, i.e., the amplitudes of the single slow waves underlying the APIA-typical steep peaks remain below the threshold of visual perception. The prerequisite for manifestation of the virtual APIA is the amplification effect, which consists of considerable stability and constant speed of an ample number (about 150) of lowvoltage potentials (of $2 \mathrm{~s}$ each) whose averaging gives rise to manifest (instead of virtual) APIAs. This is visualized within the first recording by marked steep peaks indicating APIA recorded by the electrodes T4, F8 and less marked above F7, T3, and O1; in the second recording there appears only less marked APIA at F8 and T3. The steep peaks appear in the wake of FFT-analyzed and averaged 150 consecutive $2 \mathrm{~s}$ epochs. With the first recording the electrodes T3, F8 and O1 show a noticeable multitude of peaks.

Thus, last but not least it is an amplification effect, which explains the manifestation of the virtual APIA by FFT. Amplification is out of the question with focal lesions or with heart-generated QRS-complexes and T-waves.

As already emphasized, the only possibility to avoid this artifact-type consists in excluding the APIA-prone spectral components below $2 \mathrm{~Hz}$ from any quantification procedure. In this way visual artifact-editing would become pointless because simultaneously all slow-frequent artifacts in their entirety are eliminated, in particular the loco-motor effects due to ocular- and facial muscle-activity as well as the DC-voltage generated by sweating, the QRS-complexes from ECG, the cardioballistic effect but also technical artifacts as for instance those due to sloppy fixed electrodes. For absolute certainty, one may also exclude the fronto-polar electrodes $\mathrm{Fp} 1$ and $\mathrm{Fp} 2$. We firmly hold that such a radical 


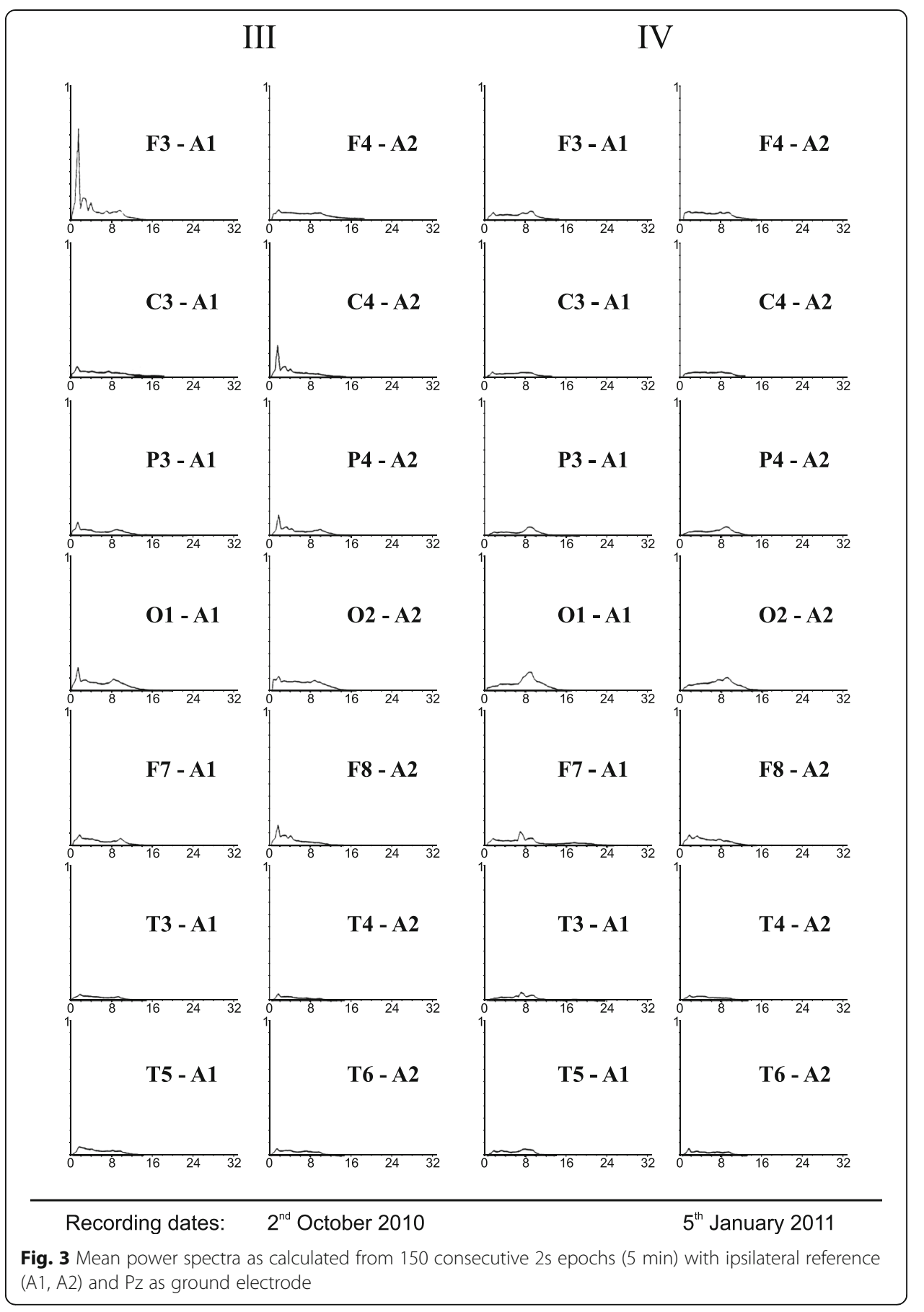

methodological realignment represents both the absolute minimum of technical expenditure as well as the optimum with regard to the attainable reliability. This is important not only to improve the potential to detect subtle EEG changes induced by psychotropic agents [5, 6] but also for elaborated approaches such as LORETA [7]. Furthermore, we hold that the exclusion of the EEG waves below $2 \mathrm{~Hz}$ and above $15 \mathrm{~Hz}$ will not have any negative impact on the information content provided by the Cerebral Global Function (CGF). Notably, the common spectral range for QEEG is arbitrary. 


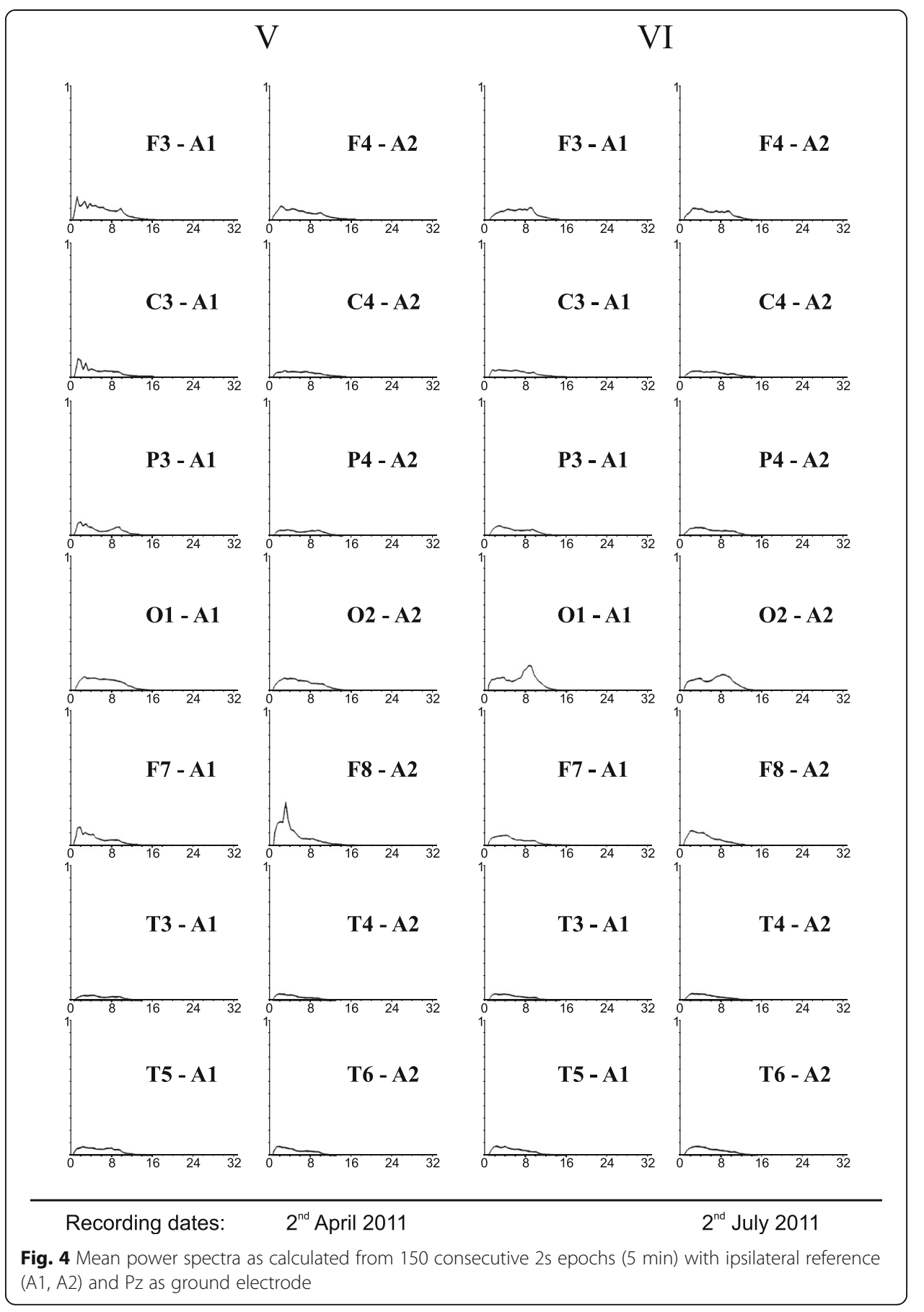

Attention should be paid to the frame of reference. This applies especially to the electrode-cap, which is being used almost exclusively today. We recapitulate that in modern EEG systems all potentials are initially recorded against one of the central reference electrodes $\mathrm{Fz}, \mathrm{Cz}$ or Pz. Thereby an advanced basis is delivered to generate typical montages such as the Common Average Reference and/or Ipsilateral Reference (to the same earlobe or mastoid). The disturbance potential of the APIA type is normally virtual, i.e. invisible. It will only become manifest (visible) by transformation of the time into the frequency- 


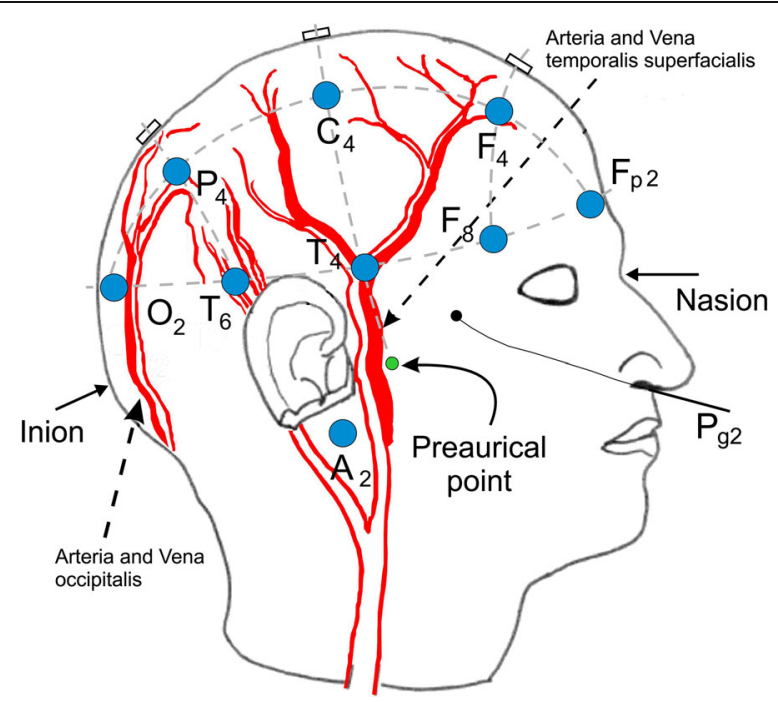

Fig. 5 Topographical relationship between electrodes and superficial head arteries

domain by means of FFT and the averaging of a certain amount of short FFTs (i.e., $>100$ $<200$ ). By generating the Common Average Reference, APIA will add up to the already addressed amplification principle (see above) and even worse therewith becomes part of the reference potential! If an ipsilateral montage is used (against the earlobe or mastoid), this will no longer make an impact on all other electrodes, as would be the case with a conventional Common Average Reference montage. Thus, one would get the subsistent APIAasymmetry. Summarized: The precondition for the demanded asymmetrical depiction in the wake of FFT is that the potentials of an ipsilateral montage (earlobe or mastoid) are fed into the FFT algorithm. In contrast, the conventional Common Average Reference without an ipsilateral montage will not show the asymmetry being typical of APIA. In this case, a deceptive bilateral-symmetric disturbance will appear.

The limitation of electrodes and therewith of spatial resolution is just the contrary to the demanded increase of up to 256 electrodes by some few authors (e.g., [8]). In the era of brain-imaging, no weighty argument suggests misusing the time-function EEG for structural diagnosis. On the other hand, the potential to objectively measure the organizational level of CGF as well as EEG-vigilance dynamics has opened new prospects for neuropsychiatric research.

Abbreviations

APIA: Arterial pulse impedance artifact; CGF: Cerebral global function; ECG: Electrocardiogram; ICA: Independent component analysis; SR-EEG: Spontaneous resting EEG

\section{Acknowledgement}

We wish to thank E. Köhler and M. Brüne for discussion of the ideas reported here as well as for support in preparing the figures. The authors report no conflict of interests. 
Authors' contribution

GU was the driving force (Spiritus rector) for the idea to publish the observations which he had already made about 20 years ago. He wrote the first draft and subsequently WS and GJ conceived the idea and revised the draft. All authors read and approved the final manuscript.

\section{Competing interests}

The authors declare that they have no competing interests.

\section{Consent for publication}

The EEG recording shown here, is that of previous coworkers who fully agreed with publication.

\section{Ethics approval and consent to participate}

This is a hypothesis paper. As explained, the EEG recordings shown here were performed from medical doctor colleagues. Due to German law an ethics approval is not necessary (see, e.g., Ethical committee of Ruhr University Bochum: http://www.ruhr-uni-bochum.de/ethik/antragsstellung.html; Hinweise 2010: Wann muss kein Antrag gestellt werden? Forschungsvorhaben, die der Qualitätssicherung dienen, ohne zusätzliche Belastungen/Untersuchungen (Engl. Translation: "When is it not necessary to apply for an ethical committee judgement? Research projects in which data are accomplished only for quality purposes without any additional harm or investigations" = this means routine diagnostic measurements performed for quality standards).

\section{Author details}

${ }^{1}$ Department of Psychiatry, Charité Benjamin Franklin, Berlin, Germany. ${ }^{2}$ Department of Psychiatry, Ruhr-University Bochum, LWL University Hospital, Bochum, Germany. ${ }^{3}$ Department of Psychiatry, Psychotherapy and Preventive Medicine, LWL University Hospital of the Ruhr University Bochum, Alexandrinenstr.1, 44791 Bochum, Germany.

Received: 17 May 2016 Accepted: 8 September 2016

Published online: 04 October 2016

\section{References}

1. Ulrich G. QUEIDA Quantitative electroencephalographic difference assessment. Hamburg: Books on Demand (BoD); 2001.

2. Ulrich $\mathrm{G}$. The theoretical interpretation of electroencephalography. The important role of spontaneous resting EEG and vigilance. Corpus Christi: BMED PRESS; 2013.

3. Ulrich G. Das spontane Ruhe-EEG. Die psychophysiologische Basis der Psychiatrie - Vergangenheit, Gegenwart und Zukunftsperspektiven. Würzburg: Königshausen \& Neumann; 2012.

4. Hjorth B. Source derivation simplifies topographic EEG interpretation. Am J EEG Technol. 1990;20:121-32.

5. Juckel G. Effekte von psychopharmaka auf das EEG. Neurophysiologie-Labor. 2002;25:89-107.

6. Juckel G, Schüle C, Pogarell O, et al. Epileptiform EEG patterns induced by mirtazapin in both psychiatric patients and healthy volunteers. J Clin Psychopharmacol. 2003;23:421-2.

7. Krause D, Folkerts $M$, Karch $S$, et al. Prediction of treatment outcome in patients with obsessivecompulsive disorder with low resolution brain electromagnetic tomography. A prospective EEG study. Front Psychol. 2016;22:6.

8. Wikswo JP, Gevins A, Williamson SJ. The future of the EEG and MEG. EEG Clin Neurophysiol. 1993;87:1-9.

\section{Submit your next manuscript to BioMed Central and we will help you at every step:}

- We accept pre-submission inquiries

- Our selector tool helps you to find the most relevant journal

- We provide round the clock customer support

- Convenient online submission

- Thorough peer review

- Inclusion in PubMed and all major indexing services

- Maximum visibility for your research

Submit your manuscript at www.biomedcentral.com/submit 